\title{
Minute-scale period oscillations of the magnetosphere
}

\author{
S. Børve ${ }^{1,3}$, H. Sato ${ }^{2}$, H. L. Pécseli ${ }^{2}$, and J. K. Trulsen ${ }^{1}$ \\ ${ }^{1}$ Institute of Theoretical Astrophysics, University of Oslo, Norway \\ ${ }^{2}$ Department of Physics, University of Oslo, Norway \\ ${ }^{3}$ Norwegian Defence Research Establishment (FFI), Norway
}

Received: 22 February 2011 - Revised: 14 March 2011 - Accepted: 25 March 2011 - Published: 13 April 2011

\begin{abstract}
Oscillations with periods on the order of 510 min have been observed by instrumented spacecrafts in the Earth's magnetosphere. These oscillations often follow sudden impacts related to coronal mass ejections. It is demonstrated that a simple model is capable of explaining these oscillations and give a scaling law for their basic characteristics in terms of the basic parameters of the problem. The period of the oscillations and their anharmonic nature, in particular, are accounted for. The model has no free adjustable numerical parameters. The results agree well with observations. The analysis is supported by numerical simulations solving the Magneto-Hydro-Dynamic (MHD) equations in two spatial dimensions, where we let a solar wind interact with a magnetic dipole representing a magnetized Earth. We consider two tilt-angles of the magnetic dipole axis. We find the formation of a magnetosheath with the magnetopause at a distance corresponding well to the analytical results. Sudden pulses in the model solar wind sets the model magnetosphere into damped oscillatory motions and quantitatively good agreement with the analytical results is achieved.
\end{abstract}

Keywords. Magnetospheric physics (Magnetospheric configuration and dynamics)

\section{Introduction}

The arrival of the pressure increase associated with the interplanetary shock driven by an interplanetary coronal mass ejection (ICME) will compress the low latitude geomagnetic field through an intensification of the Chapman-Ferraro magnetopause current. This leads to a sudden impulse (SI) which can be observed also in low latitude magnetometer records.

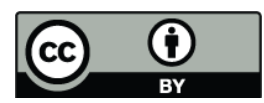

Correspondence to: H. L. Pécseli

(hans.pecseli@fys.uio.no)
In a recent publication (Farrugia and Gratton, 2011) it was demonstrated that such SI-events are followed by large amplitude oscillations of $\sim 5$ min periods. These are observed, for instance, by satellites in the cold, dense magnetosheath and in the hot and tenuous magnetosphere plasmas, consistent with other related observations (Plaschke et al., 2009). It has also been found (Kivelson et al., 1984; Sibeck et al., 1989; Korotova and Sibeck, 1995) that magnetic pulsations with $8-10$ min periods measured by geosynchronous satellites are well correlated with oscillations in the solar wind dynamic pressure.

It is the purpose of this communication to demonstrate that oscillations at these characteristic periods can be accounted for by a simple model of the magnetosphere. The entire problem of the coupling between the solar wind and the magnetosphere is extremely complicated even under quiet conditions, and will be even more involved during solar wind disturbances. The main purpose of the present work is to reduce the analysis to its bare essentials, and then compare the results with observations and numerical simulations. The present approach is global, while some local models (Samson et al., 1992) study field line resonances associated with Magneto-Hydro-Dynamic (MHD) waveguide modes in the magnetosphere. Other models consider waves propagating in the equatorial plane between the flanks of the bow shock and a turning point deep within the magnetosphere (Harrold and Samson, 1992). Another approach considers the magnetopause surface analogous to an elastic membrane, obtaining its natural modes of oscillation (Freeman et al., 1995).

\section{A simple model problem}

Assume as a first approximation that the solar wind can be considered as a "wall" of ideally conducting material. Surface currents are induced in the solar wind, in such a way that the Earth's dipolar magnetic field together with the magnetic 


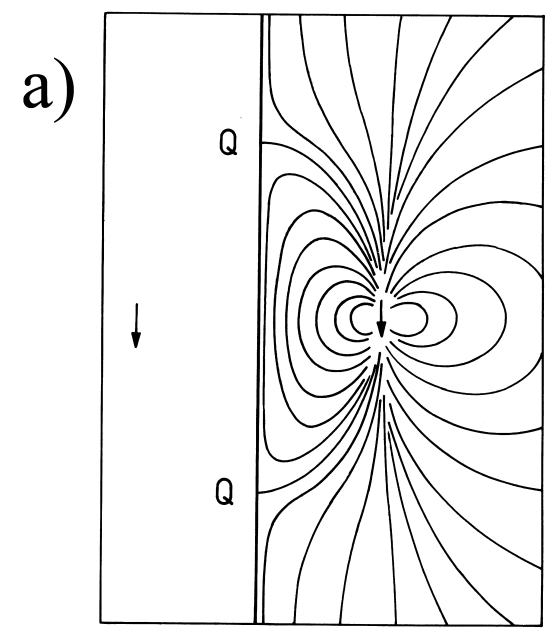

b)
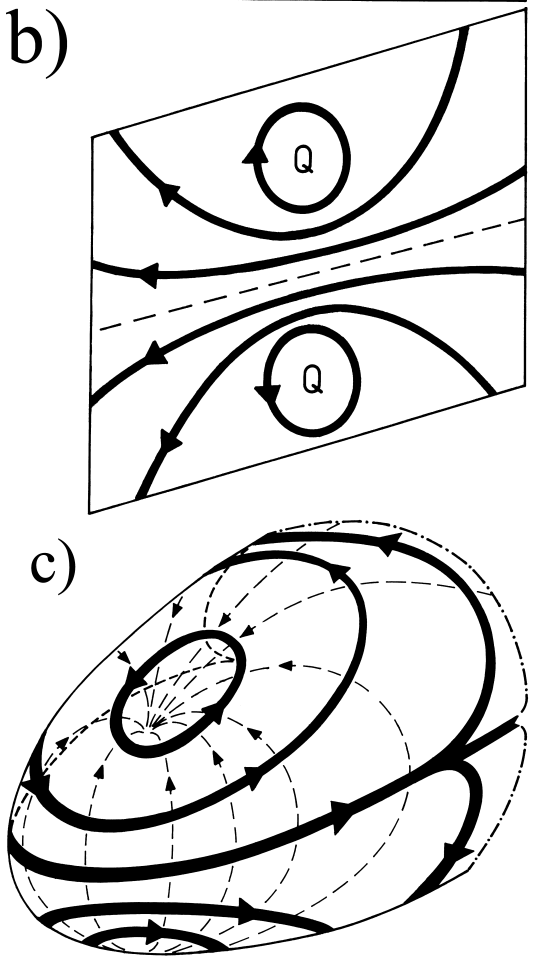

Fig. 1. Simple illustrative model for the magnetosphere, obtained by considering the solar wind as an ideally conducting wall (Chapman and Bartels, 1940; Alfvén, 1950). (a) shows the magnetic field lines, and (b) selected surface current paths at the interface, being representative for a continuous distribution. The magnetic field vanishes at the cusp-points labeled $Q$. A schematic illustration of the deformation of the surface of current-paths in (b) is shown in (c) for a more realistic model.

fields originating from the surface currents cancel inside the model solar wind. This situation is illustrated in Figs. 1a and 1b. For a stationary observer it will appear as if the magnetic field lines near the Earth are "compressed". The magnetic field between the Earth and the ideal solar wind can be determined by the method of images, where an image magnetic dipole is placed inside the solar wind, as indicated by the arrow to the left in Fig. 1a. We will not need this exact solution here, but be content with the overall variation. Note the two cusp points labeled $Q$ on the figure, where the magnetic field intensity vanishes. Using the mirror image and the basic expressions for a magnetic dipole, the construction of Fig. 1a is straight forward. For simplicity we let the magnetic dipole be parallel to the surface of the interface in Fig. 1a, this is a trivial restriction.

The plane surface approximation is only locally valid: the surface containing the current paths is distorted as illustrated in Fig. 1c). The topology of the surface currents is however not changed. The plane surface model can therefore be used as an approximation as the tangent plane at the stagnation point (or "nose region") of the solar wind. The model assumes an ideally conducting solar wind. For large magnetic Reynolds' numbers $R_{\mathrm{L}} \equiv \mu_{0} \sigma \mathcal{L} U \gg 1$ the assumptions are applicable even for finite conductivities $\sigma$, with $\mathcal{L}$ being a characteristic length-scale for the problem. In our case we estimate $R_{\mathrm{L}} \approx 10^{8}$. A small magnetic field of $\sim 5 \mathrm{nT}$ embedded in the solar wind is of no significant consequence for the arguments, and will only change the estimate for $R_{\mathrm{L}}$ slightly.

\subsection{Steady state}

We can use the simplified model from Fig. 1 to obtain an estimate for the distance $R$ from the Earth to the stagnation point between the Earth and the Sun (Walker and Russell, 1995). We take the dipolar Earth magnetic field component $B_{\theta}=\mu_{0} \mathcal{M} \sin \theta /\left(4 \pi r^{3}\right)$ and derive the magnetic field pressure $B^{2} / 2 \mu_{0}$ at this position. An angle $\theta$ between the magnetic dipole axis and the Sun-Earth direction was introduced explicitly, noting that for most relevant cases we have $\theta \approx \pi / 2$. With the additional magnetic field contribution from the image dipole, see Fig. 1, we find $B^{2} / 2 \mu_{0}=$ $2 \mu_{0} \mathcal{M}^{2} \sin ^{2} \theta /\left(4 \pi r^{3}\right)^{2}$. For stationary conditions, this magnetic pressure has to balance the dynamic pressure from the solar wind. With this latter pressure being the momentum received per sec per unit area, we have the estimate $p=U^{2} M n$. We used only the directed momentum density of the solar wind $n M U$, with $M$ being an average ion mass, and ignored a thermal velocity spread. This can be justified since $U$ is large compared to the sound speed $C_{s}$ as well as the ion thermal velocity. The net force per unit area on the magnetopause is then

$F=2 \frac{\mu_{0} \mathcal{M}^{2} \sin ^{2} \theta}{\left(4 \pi r^{3}\right)^{2}}-n M U^{2}$,

where $r$ is a distance in the Earth-Sun direction, as measured from the Earth. For $\theta \approx \pi / 2$ we note that the correction due to the tilt of the magnetic dipole is of the order of $(\pi / 2-\theta)^{2}$. This correction is small and will be ignored in the following. 


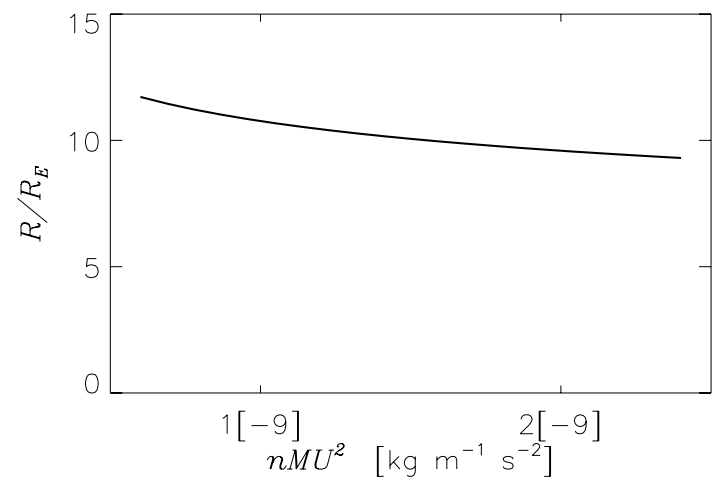

Fig. 2. Normalized Earth-magnetopause distance $R / R_{\mathrm{E}}$ for varying dynamic solar wind pressure $n M U^{2}$.

The equilibrium position $R$ is found by equating the magnetic and solar wind pressures. We find the relation

$R=\left(\frac{\mu_{0} \mathcal{M}^{2}}{8 \pi^{2} n M U^{2}}\right)^{1 / 6}$

Similar expressions can be found in the literature (Walker and Russell, 1995). Inserting typical numbers as $U \approx 3 \times$ $10^{5} \mathrm{~m} \mathrm{~s}^{-1}, n \approx 5 \times 10^{6} \mathrm{~m}^{-3}$, and the hydrogen mass, $M=$ $1.66 \times 10^{-27} \mathrm{~kg}$, we find $R \approx 7.2 \times 10^{7} \mathrm{~m}$, or $R \approx 11.2 R_{\mathrm{E}}$, in terms of the Earth radius, $R_{\mathrm{E}}=6.4 \times 10^{6} \mathrm{~m}$. The estimate for $R$ is comfortably close to the generally accepted range of $R \sim 10-15 R_{\mathrm{E}}$. The model Eq. (2) implies a scaling law for the distance to the magnetosheath boundary in terms of the solar wind velocity $U$ and the solar wind mass density $n M$. Note that there are no free parameters to fit in Eq. (2).

The numerical values chosen here are somewhat ad-hoc. To illustrate the robustness of the results, we show in Fig. 2 the variability of the normalized Earth-magnetopause distance $R / R_{\mathrm{E}}$ for varying solar wind pressures $n M U^{2}$. We find that the good agreement with known observational results is robust. It is therefore reasonable to explore also the dynamical properties of this simple model, its natural oscillation period in particular.

\subsection{Oscillations without damping}

The model discussed here allows for oscillations of the magnetopause around the equilibrium position $R$. For small, slow displacements of the interface between the solar wind and the magnetosphere, we can assume the solar wind pressure to be constant, while the magnetic pressure varies like $1 / r^{6}$. Assuming small displacements $\Delta$ from the equilibrium position $R$, we will consequently have a net force on the interface given approximately by $F \approx-3 \Delta \mu_{0} \mathcal{M}^{2} /\left(4 \pi^{2} R^{7}\right)$. To set up an equation of motion we introduce the mass loading (i.e.

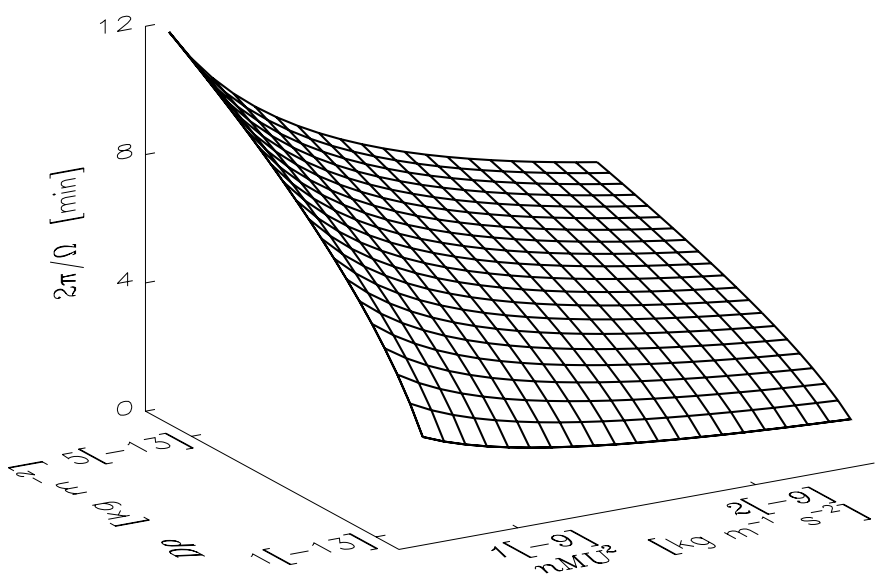

Fig. 3. The period $2 \pi / \Omega$ of characteristic small amplitude magnetosphere boundary oscillations for varying solar wind pressure $n M U^{2}$ and mass loading $D \rho$.

mass per unit surface area) of the magnetopause, here written as the product of a thickness $D$ and a mass density $\rho$ to find

$D \rho \frac{d^{2} \Delta}{d t^{2}}=-3 \Delta \frac{\mu_{0} \mathcal{M}^{2}}{4 \pi^{2} R^{7}}$,

giving the oscillation period

$T=\frac{2 \pi}{\Omega}=2 \pi \sqrt{\frac{4 \pi^{2} R^{7} D \rho}{3 \mu_{0} \mathcal{M}^{2}}}=2 \pi \frac{R}{U} \sqrt{\frac{D \rho}{6 n M R}}$,

using Eq. (2). For simplicity we assume here that the mass density $\rho$ is approximately uniform, while in reality there can be some irregular variations (Song et al., 1990; Gosling et al., 1990).

To estimate the mass loading $D \rho$ giving the inertial term in Eq. (3) we use results from (Spreiter et al., 1966). The importance of the inertia for the problem was recognized also in other studies (Smit, 1968; Freeman et al., 1995). The numerical studies (Spreiter et al., 1966) indicate that typical values are $D \approx R / 4$ (consistent with observations by e.g. Song et al., 1990) and $\rho \approx 4 n M$. As long as the velocity of the oscillations $d \Delta / d t$ is smaller than the speed of sound, $C_{\mathrm{s}}$, we can consider the motion to be incompressible (Landau and Lifshitz, 1987), and therefore let $D \rho$ be constant. (It is easily demonstrated that $d \Delta / d t / C_{\mathrm{s}}$ can become large only for large disturbances. In effect, we assume only that $D \rho$ is constant, which is a weaker assumption than strict incompressibility, $\rho \approx$ const.). Inserting the numerical values used before into the analytical result (4) we find a characteristic period to be $2 \pi / \Omega=10.2 \mathrm{~min}$, which is close to those observed. Within the present model the magnetosphere can be considered as an oscillator which is set into a "ringing" motion by a sudden impulse event. The oscillations are global, and will give detectable signatures also in ground-based instruments measuring magnetic fields, as observed. 
In Fig. 3 we illustrate the period of the characteristic magnetosphere boundary oscillations for varying solar wind pressure $n M U^{2}$ and mass loading $D \rho$ as defined before. We find characteristic periods in the interval 2-12 min (corresponding to the frequency range $1-8 \mathrm{mHz}$ ), which accommodate observations very well (Plaschke et al., 2009). Also we find the results to be robust in the sense that even large variations in one of the parameters only give modest changes in $2 \pi / \Omega$. A change in solar wind momentum density changes the equilibrium position, and we have $\Delta R / R \approx$ $-(1 / 6) \Delta(n M U) /(n M U)$. We emphasize that there are no free parameters to fit in Eq. (3). We use quantities such as $D$ and $\rho$ as inputs, but note that all quantities are amenable to measurements or numerical simulations, so they can not properly be considered as free parameters available for fitting analytical results to observations. Our results in the present work can be seen as an effort to predict some dynamic properties of magnetospheres, on the basis of measurable steady state characteristics.

The simple model outlined here has several features that can be tested experimentally. Due to the strongly anharmonic nature of the restoring force we expect a significant harmonic content. Also the oscillations should have a detectable nonlinear frequency shift.

To discuss the finite amplitude nonlinear case, we rewrite the force without linearization to obtain Newton's second law in the form

$D \rho \frac{d^{2}}{d t^{2}} \Delta=-\frac{\mu_{0} \mathcal{M}^{2}}{8 \pi^{2} R^{6}}\left(1-\frac{1}{(1+\Delta / R)^{6}}\right)$,

which gives Eq. (3) upon linearization of the right hand side. Introducing the frequency $\Omega$ of the small amplitude oscillations we can write Eq. (5) as

$$
\frac{d^{2}}{d t^{2}}\left(\frac{\Delta}{R}\right)=-\frac{\Omega^{2}}{6}\left(1-\frac{1}{(1+\Delta / R)^{6}}\right)
$$

or

$$
\left(\frac{d Z}{d t}\right)^{2}+\frac{\Omega^{2}}{3}\left(Z+\frac{1}{5(1+Z)^{5}}-\frac{1}{5}\right)=\Omega^{2} A^{2}
$$

with $Z \equiv \Delta / R$ and the right hand side being an integration constant, written in this form for later convenience. The quantity $R \Omega A$ is the velocity of the perturbation at the equilibrium position. By integration we obtain the oscillation period

$T=\frac{\sqrt{3}}{\Omega} \int_{Z_{1}}^{Z_{2}} \frac{d Z}{\sqrt{3 A^{2}-Z-\frac{1}{5(1+Z)^{5}}+\frac{1}{5}}}$,

where the integration limits $Z_{1}<0$ and $Z_{2}>0$, with $\left|Z_{1}\right| \neq$ $\left|Z_{2}\right|$, are given as the solutions of $Z-1 / 5+1 /\left(5(1+Z)^{5}\right)=$ $3 A^{2}$. The amplitude dependence of the normalized variation of the oscillation period is shown in Fig. 4. The nonlinear frequency shift is significant, being up to $\sim 10 \%$, and should be observable. The anharmonic features can be made even
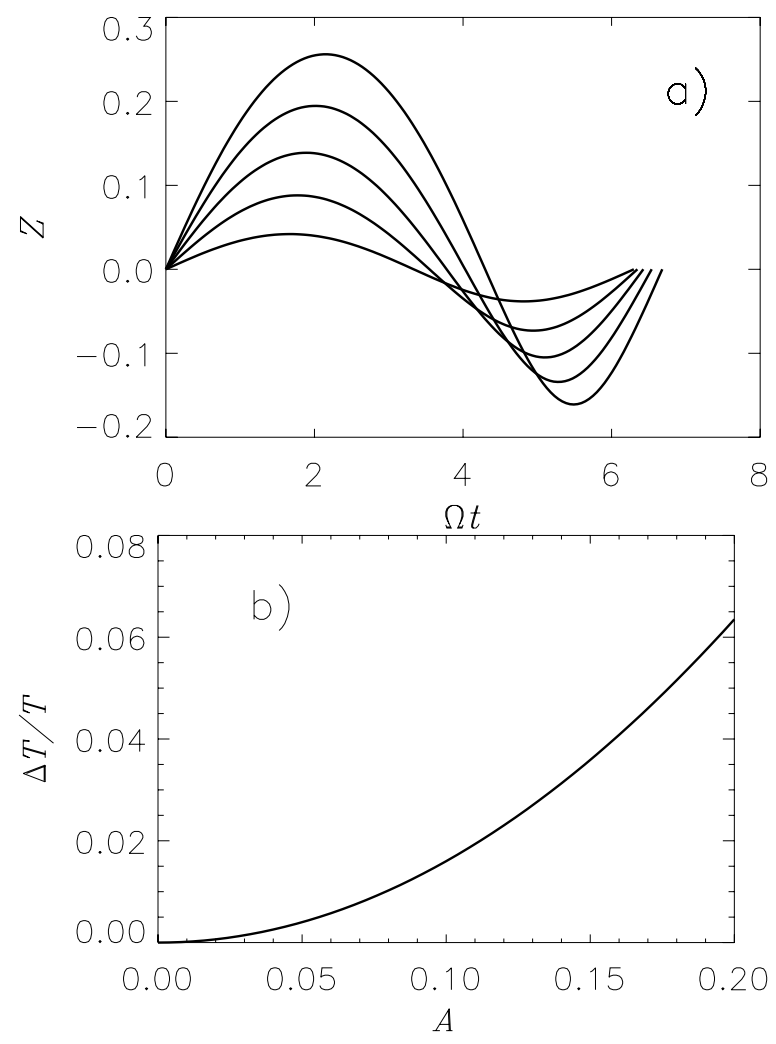

Fig. 4. Numerical solutions of Eq. (5) are shown in (a) for five normalized amplitudes $A=0.04,0.08,0.12,0.16,0.20$. The normalized nonlinear frequency shift of the characteristic magnetospheric boundary oscillations shown in (b) for varying $A$, where $A R \Omega$ is the reference velocity $d \Delta / d t$ at the position $\Delta=0$.

more conspicuous by considering the velocity and acceleration of the boundary layer, but these quantities can not be detected experimentally, so they are not shown here. We can also demonstrate by a simple Fourier analysis that the oscillations will have a rich harmonic content even for moderate oscillation amplitudes due to the strongly anharmonic nature of the restoring force in Eq. (5). The amplitude of the harmonics is increasing with amplitude $A$. Harmonics of the magnetospheric oscillations are often observed (Kepko and Spence, 2003).

\subsection{Damped oscillations}

The oscillations observed in space are often strongly damped, in variance with the simple model discussed in Sect. 2.2. The following extension of our basic model will account also for a damping mechanism when we take into account that the momentum transferred from the solar wind to the magnetosphere is determined by the relative velocities, and not by $U$ alone as assumed in Sect. 2.2. The more general expression for the force (1) can be written as 


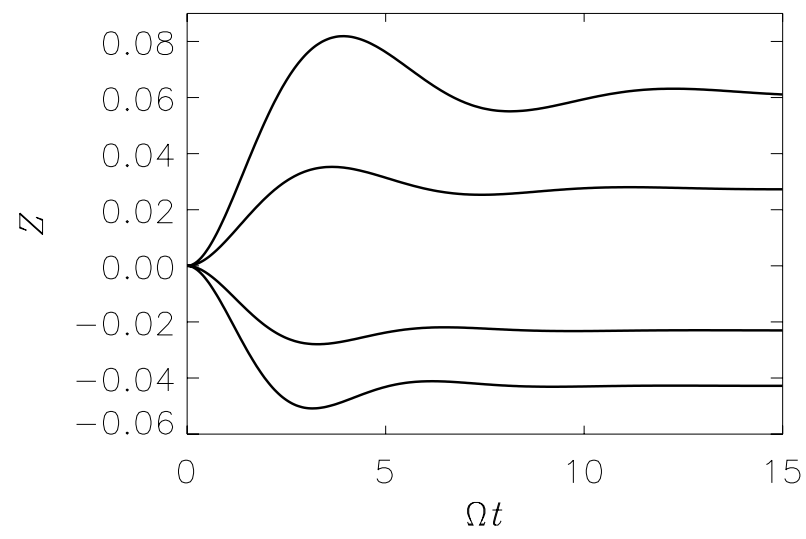

Fig. 5. Numerical solutions of Eq. (9) are shown for four amplitudes $\gamma= \pm 0.15$ and \pm 0.30 .

$F=2 \frac{\mu_{0} \mathcal{M}^{2} \sin ^{2} \theta}{\left(4 \pi(R+\Delta)^{3}\right)^{2}}-n M\left(U+\frac{d \Delta}{d t}\right)^{2}$.

Taking again $\theta \approx \pi / 2$, expression (5) becomes

$$
\begin{aligned}
D \rho \frac{d^{2}}{d t^{2}} \Delta= & -\frac{\mu_{0} \mathcal{M}^{2}}{8 \pi^{2} R^{6}}\left(1-\frac{1}{(1+\Delta / R)^{6}}\right) \\
& -n M\left(2 U \frac{d \Delta}{d t}+\left(\frac{d \Delta}{d t}\right)^{2}\right) .
\end{aligned}
$$

In the normalized units used before we have

$$
\begin{aligned}
\frac{d^{2}}{d \tau^{2}} Z= & -\frac{1}{6}\left(1-\frac{1}{(1+Z)^{6}}\right) \\
& -\frac{2}{\sqrt{6}} \sqrt{\frac{R n M}{D \rho}} \frac{d Z}{d \tau}-\frac{R n M}{D \rho}\left(\frac{d Z}{d \tau}\right)^{2},
\end{aligned}
$$

in terms of the normalized time $\tau \equiv \Omega t$. We linearize Eq. (9) to obtain the result

$\frac{d^{2} Z}{d \tau^{2}}=-Z-2 \alpha \frac{d Z}{d \tau}$,

with the normalized damping coefficient $\alpha \equiv \sqrt{R n M / 6 D \rho}$. The result (10) has well known solutions in form of damped oscillations $Z(\tau)=\cos (\omega \tau+\delta) \exp (-\tau \alpha)$ with $\omega \equiv \sqrt{1-\alpha^{2}}$ when $\alpha<1$. For $\alpha=1$ we have critical damping, while $\alpha>1$ gives over-damped oscillations. Small values of $\alpha$ are found when the solar wind speed $U$ is large (giving small $R$ ) and the mass loading $D \rho$ is large as well. For most relevant cases we have $\alpha<1$ but the damping of the oscillations may nonetheless be strong, so that nonlinear effects will be noticeable only for the initial part of the time evolution of a disturbance. The numerical example used in Sect. 2.2 had $R n M=D \rho$ giving $\alpha=1 / \sqrt{6} \approx 0.41$.

A relevant problem to be analyzed by Eq. (9) corresponds to a sudden enhancement of the solar wind plasma density, which we here model by increasing $n M$ while keeping $U$ constant. We use the unperturbed condition for the normalizing quantities and let $\gamma$ be the fraction of solar wind mass density enhancement. The basic equation can then be written in normalized form as

$$
\frac{d^{2} Z}{d \tau^{2}}=\frac{1}{6} \frac{1}{(1+Z)^{6}}-\frac{1}{6}\left(1+\sqrt{\frac{6 R n M}{D \rho}} \frac{d Z}{d \tau}\right)^{2}(1+\gamma) \text {. }
$$

In Fig. 5 we show numerical solutions of Eq. (11) for different perturbations $\gamma$ to illustrate the damping of the oscillations. This reference calculation uses $R n M=D \rho$. To illustrate the nonlinear character of the oscillations, we show solutions for both positive and negative changes in the solar wind density. For a linear system, the positive and negative parts of Fig. 5 should be mirror images with respect to the horizontal axis. We expect, however, a different nonlinear response to an increase and a rarefaction in the solar wind. We find that the term containing $(d Z / d t)^{2}$ reduces the damping slightly for realistic amplitudes.

The physical mechanism causing the damping in Eq. (10) is seen to be a phase-lag between the forcing and the displacement of the magnetospheric boundary when it is taken into account that the momentum transfer depends on the solar wind velocity relative to the moving boundary.

\section{Numerical simulations}

In order to make a qualitative test of the foregoing simple models we carried out some numerical simulations of the interaction of a Solar wind and a magnetic dipole representing the Earth. For simplicity, our simulations are carried out in two spatial dimensions. In this representation, the Earth's magnetic field is modeled not by a small ring current but by two parallel wires, carrying current in opposite directions, perpendicular to the plane of computation. The analytical model refers to the dynamics of the tangent plane at the stagnation point for the solar wind. This plane can be defined for three as well as two-dimensional conditions, but the analytical expressions are slightly different for the two cases.

The numerical methods used are based on a SmoothParticle-Hydrodynamics (SPH) code solving the MagnetoHydro-Dynamic (MHD) equations (Monaghan, 1992, 2005; Børve et al., 2005). To generalize the analytical results, we allow the Solar wind to support a weak magnetic field. In this two-dimensional representation, the dipole field becomes

$\mathbf{B}(\mathbf{r})=B_{\mathrm{E}}\left(\frac{R_{\mathrm{E}}}{r}\right)^{2}\left(\cos \theta \widehat{\mathbf{e}}_{\theta}-\sin \theta \widehat{\mathbf{e}}_{r}\right)$

in terms of a reference magnetic field $B_{\mathrm{E}}$ at a reference distance $R_{\mathrm{E}}$. We note that for this 2-D-model, the magnetic field intensity $|B|$ is independent of $\theta$. We can write the equivalent of Eq. (8) in the form 


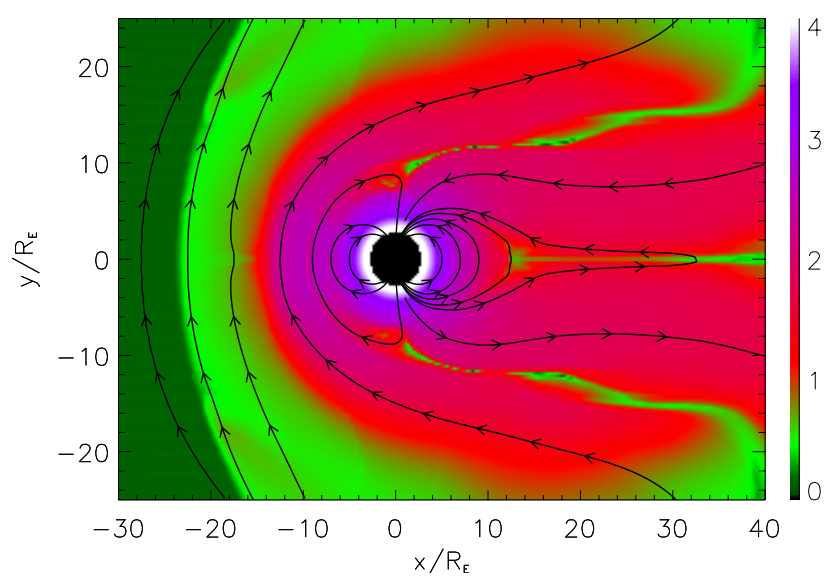

Fig. 6. The magnetic field intensity, here represented by $\ln (|B|+1)$, is shown in color coding with selected magnetic field lines superimposed. Distances are normalized with the Earth radius $R_{\mathrm{E}}$. See corresponding Fig. 7 for the plasma density. The Sun is in the negative $\mathrm{x}$-direction.

$$
\begin{aligned}
D \rho \frac{d^{2}}{d t^{2}} \Delta= & \frac{2 B_{\mathrm{E}}^{2}}{\mu_{0}}\left(\frac{R_{\mathrm{E}}}{R}\right)^{4} \frac{1}{(1+\Delta / R)^{4}} \\
& -n M\left(U+\frac{d \Delta}{d t}\right)^{2},
\end{aligned}
$$

where the equilibrium position is

$$
R=R_{\mathrm{E}}\left(\frac{2 B_{\mathrm{E}}^{2}}{\mu_{0} n M U^{2}}\right)^{1 / 4},
$$

and the characteristic oscillation period

$$
\frac{2 \pi}{\Omega}=\frac{2 \pi R^{5 / 2}}{B_{\mathrm{E}} R_{E}^{2}} \sqrt{\frac{\mu_{0} D \rho}{8}}=2 \pi \frac{R}{U} \frac{1}{2} \sqrt{\frac{D \rho}{R n M}} .
$$

A change in solar wind momentum density changes the equilibrium position, and in the present two-dimensional model we have $\Delta R / R \approx-(1 / 4) \Delta(n M U) /(n M U)$.

In normalized units, the expression (13) becomes

$$
\begin{aligned}
\frac{d^{2}}{d \tau^{2}} Z= & -\frac{1}{4}\left(1-\frac{1}{(1+Z)^{4}}\right) \\
& -\sqrt{\frac{R n M}{D \rho}} \frac{d Z}{d \tau}-\frac{R n M}{D \rho}\left(\frac{d Z}{d \tau}\right)^{2} .
\end{aligned}
$$

The nonlinear features are less prominent in two spatial dimensions, as seen by comparing the nonlinear term $(1+Z)^{-4}$ in Eq. (15) with $(1+Z)^{-6}$ in Eq. (9). The present twodimensional results are, however, not significantly different from the model outlined in Sect. 2. Our model is thus robust, and can be tested also with a simplified two-dimensional numerical model as the one used here.

Representative results are shown in Fig. 6 showing the magnetic field intensity represented by $\ln (|B|+1)$ in color

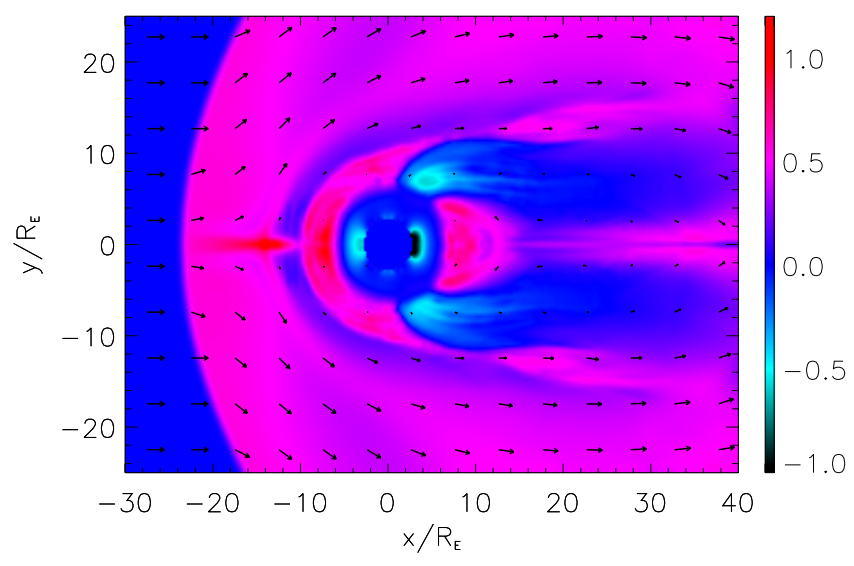

Fig. 7. Variation of the plasma density, here represented by $\log _{10}(n)$, is shown with velocity vectors superimposed. See corresponding Fig. 6 for the magnetic field.

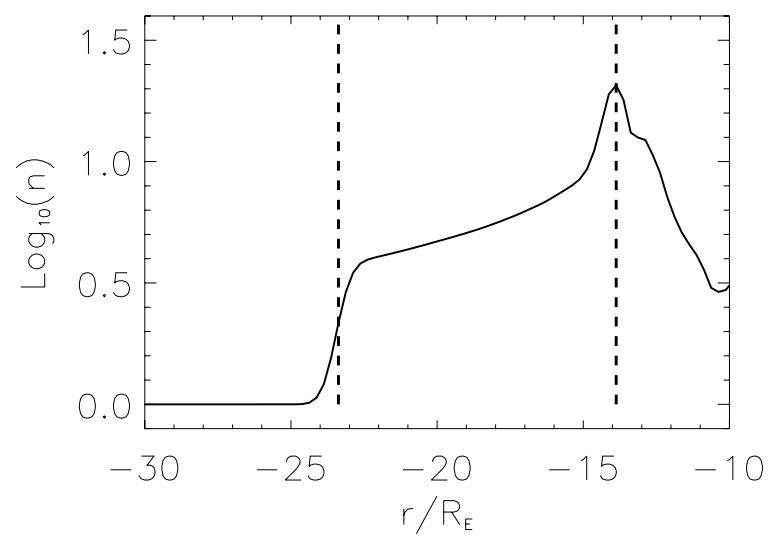

Fig. 8. Variation of plasma density along the line connecting the Sun and the Earth. Distance is normalized also here with the Earth radius $R_{\mathrm{E}}$. This figure serves to define the positions (shown with vertical dashed lines) of the bow shock (left) and the magnetopause (right) as used later on. The density is normalized by the steady state solar wind plasma density. Note the logarithmic vertical axis. Even small variations of the density can give a large change in the local maximum to the right, so motions of this position are not always well defined.

coding with selected magnetic field lines superimposed, while Fig. 7 shows the plasma density by $\log _{10}(n)$ with solar wind velocity vectors superimposed. Note the ring-shaped magnetic field intensity near the Earth in Fig. 6, consistent with Eq. (12). A part of the axial variation of the plasma density is shown in Fig. 8. For the numerical results in Figs. 6-8 the positive $\mathrm{X}$-axis (with origin at the Earth) is pointing away from the Sun. The magnetosheath plasma density is larger than in a fully three-dimensional case since the plasma can escape from the stagnation point in two directions only. The distance from Earth to the magnetosheath is approximately $R=14 R_{\mathrm{E}}$, in reasonable agreement with predictions of our model. 


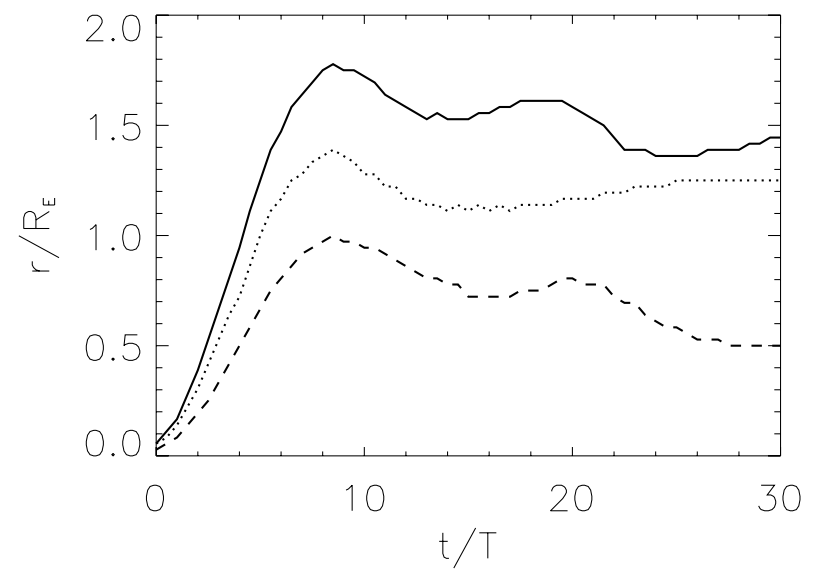

Fig. 9. Bow shock boundary oscillations, see Fig. 8 for definition of the position being considered. The normalizing time is here $T \equiv R_{\mathrm{E}} / U$ where $U$ is the solar wind velocity. We show results for $\Delta n / n=10 \%, 15 \%$ and $20 \%$, keeping the solar wind velocity constant. See also Fig. 10.

When this reference model has reached a steady state, we impose a sudden increase $\Delta n$ in the solar wind density. The two positions indicated in Fig. 8 are then set in motion. A mechanical equivalent could be a damped spring at rest with a mass load $M$ which at $t=0$ is hit inelastically by a projectile with mass $\Delta M$, and carrying momentum $U \Delta M$. This spring will be set into oscillatory motion until the entire system settles at its new equilibrium state. In Figs. 9 and 10 we show the time variations of the positions of the bow shock and the magnetopause as defined in Fig. 8. The numerical simulations give a smooth density variation between the two boundaries shown in Fig. 8, while observations show irregularities in the density. These probably originate from density variation in the solar wind. The density profile in the simulations changes significantly during the dynamical evolution following the perturbation, so the motion of the local maximum position at the inner boundary at the magnetopause (right hand position indicated in Fig. 8) is not always well defined.

We observe that the magnetosphere is set into damped oscillations, starting at the reference position, oscillating to eventually settle at the new equilibrium position consistent with the new (increased) solar wind pressure. The two positions indicated in Fig. 8 move together in phase, albeit with different amplitudes. We find $\sqrt{R n M / D \rho} \approx 0.5$. The oscillation period is found to be of the order of $11 R_{\mathrm{E}} / U \approx$ $(11 / 14) R / U$. This result is within an order of magnitude consistent with Eq. (14), which predicts a period of approximately $2 \pi / \Omega=2 \pi R / U$ for the present conditions. The oscillations are damped, with a damping time of 1-2 oscillation periods. From the numerical results we estimate $\alpha=\sqrt{\operatorname{RnM}(4 D \rho)} \approx 0.25$, in reasonable agreement with the observed damping time. A nonlinear frequency shift is here barely noticeable, in agreement with the properties of

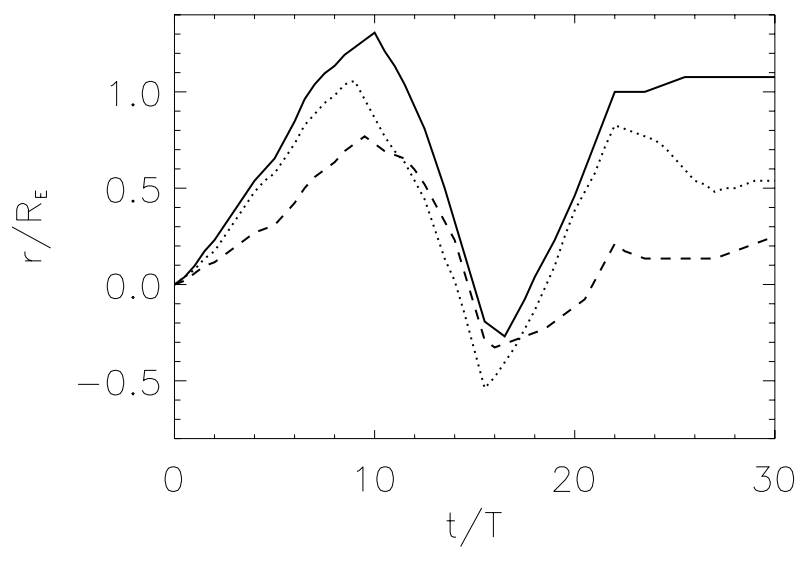

Fig. 10. Magnetopause boundary oscillations corresponding to Fig. 9. See Fig. 8 for definition of the position considered.

the present two-dimensional model. The results are thus in qualitative agreement with the analytical results of Sect. 2.3 and Fig. 5.

\subsection{Simulations with a tilted magnetic dipole}

Most of the analysis and the numerical simulations presented so far refer to the case where the magnetic dipole axis is perpendicular to the direction from the Sun to the Earth. The analytical expression (1) allows for a tilt of the magnetic dipole, so formally this simplifying assumption can be relaxed, but the complexity of the problem becomes significantly increased, nonetheless. We can use the numerical simulations to estimate the significance of the assumption of $\theta=\pi / 2$. Numerical results are shown in Figs. 11 and 12, to be compared with Figs. 6 and 7 for the magnetic field and density variations, while the time-variations shown in Fig. 13 should be compared with Figs. 9 and 10. We find that all the basic features of the simplified $\theta=\pi / 2$ model are recovered, the main difference being that a noticeable phase shift between the oscillating spatial displacement of the two boundaries defined on Fig. 8. We also find a reduction in the equilibrium distance $R$, consistent with Eq. (2) if a $\sin ^{2} \theta$ correction is included. The average plasma density of the magnetosheath in the simulations is found to decrease with 15-20\% when the dipole axis is tilted, while the width $D$ changes only little. The characteristic period of the oscillations is slightly reduced as compared to the case without tilt of the magnetic axis, and the damping time is slightly increased, so that also this observation is in qualitative agreement with our analytical model.

\section{Conclusions}

We have described a simple model that takes into account the basic features of the interface between the Earth's magnetosphere and the solar wind. We demonstrated how this simple 


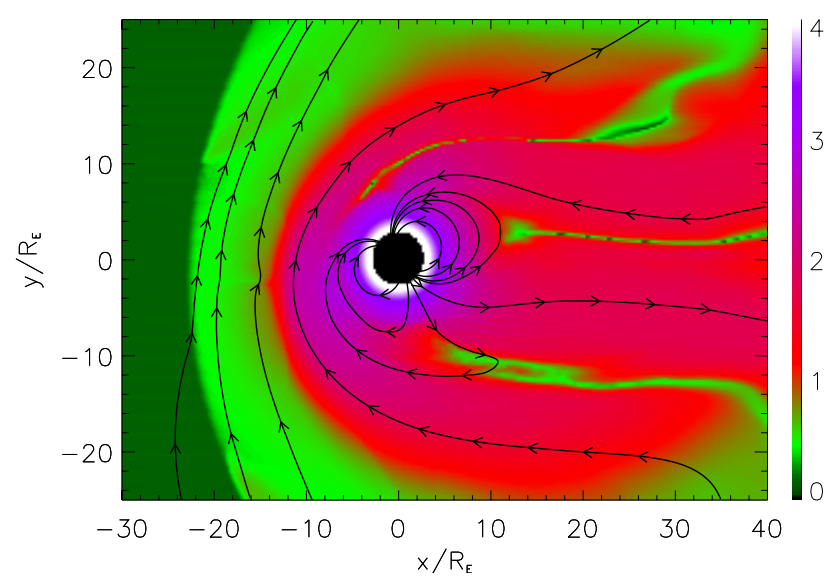

Fig. 11. Magnetic field variations, corresponding to Fig. 6. The present case has a tilt of $\pi / 6$ for the magnetic dipole axis.

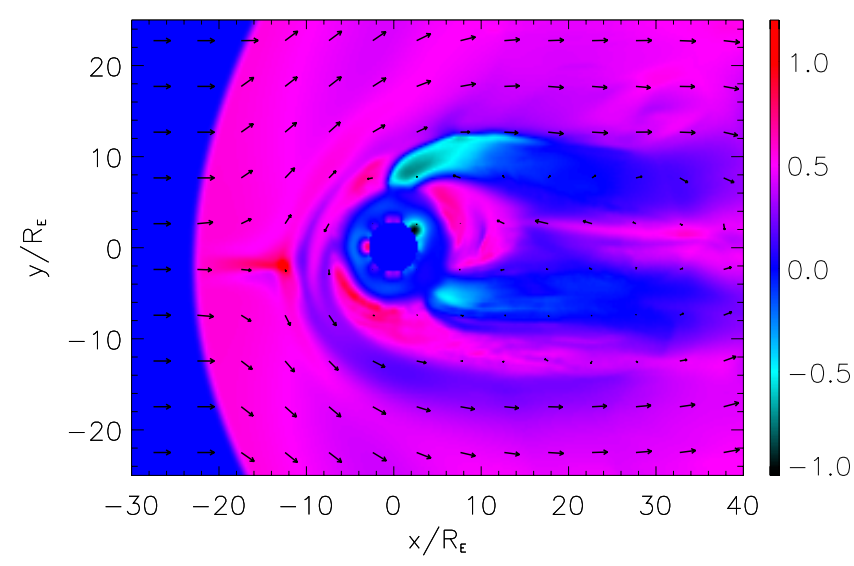

Fig. 12. Density variations corresponding to Fig. 7, here with a tilt of $\pi / 6$ for the magnetic dipole axis.

model accounts for the basic characteristics of the distance from the Earth to the magnetosphere boundary and also how the same model accounts for observed characteristic minute scale oscillations often observed in the magnetosphere following SI-events. The observed periods of oscillation can be accounted for. It is also shown how a simple extension of the model explains the damping of the oscillations. Nonlinear effects were included in the analysis, and these can have importance for cases with weakly damped oscillations. We believe that the suggested model can be applied also to other magnetized planets in a solar system.

In support of the analytical model we show results from numerical simulations, obtained for a model system in two spatial dimensions. Good qualitative agreement is found. A sudden change in the solar wind momentum density gives rise to damped oscillations of the boundaries of the computational magnetopause. The simulations demonstrate that the solar wind magnetic field has only minor importance. Its presence will reduce the net magnetic pressure differ-
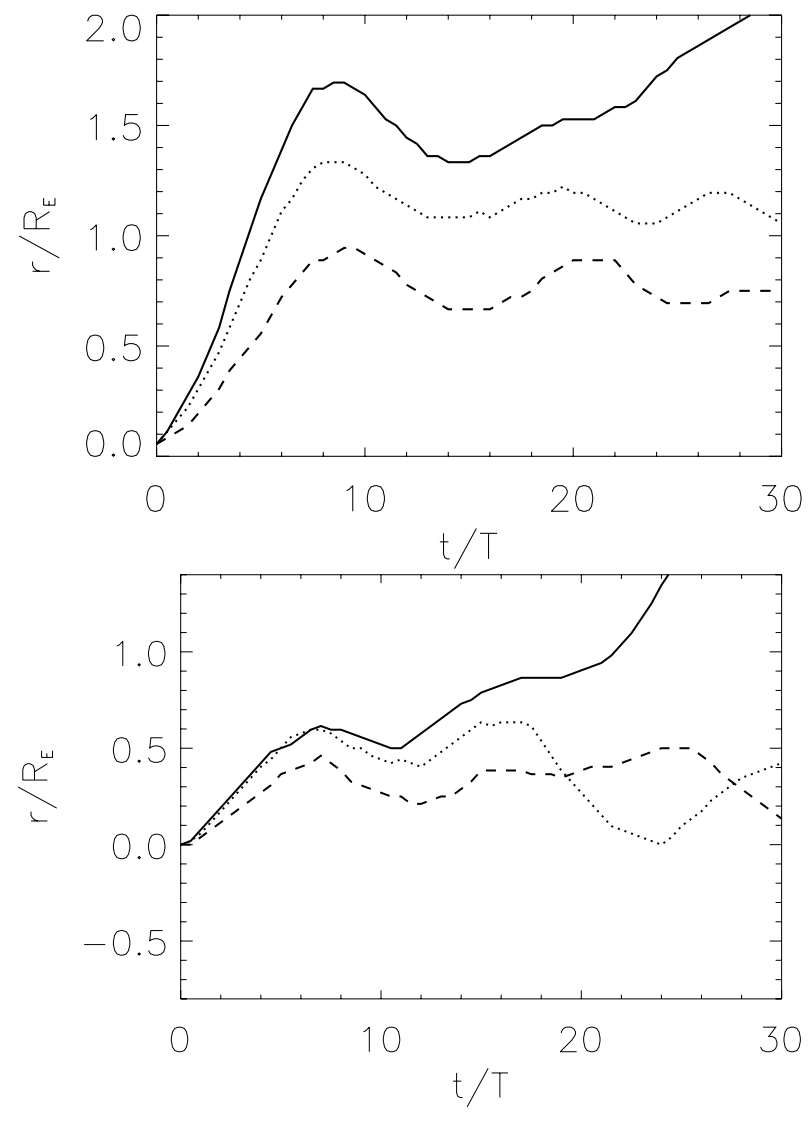

Fig. 13. Figures corresponding to Figs. 9 (top) and 10 (bottom).

ence across the magnetospheric boundary as compared to our model and thus reduce the oscillation frequency. The different amplitudes of the oscillations of the bow shock and the magnetosphere boundary in Figs. 9 and 10 indicate that a more accurate analysis should take into account the compressibility of the magnetospheric plasma, i.e. take into account the time it takes for the perturbation to propagate from the bow shock to the magnetosphere boundary. The relatively strong damping in our simulations makes the nonlinear frequency shift barely noticeable, they might be observable for weaker dampings, i.e. smaller values of $\sqrt{R n M / 6 D \rho}$.

In the small amplitude limit we have a normalized damping constant $\alpha=\sqrt{R n M / 6 D \rho}$ which has to be determined by observations or numerical simulations. The damping is very sensitive to changes in $\alpha$. Quite generally we can state that $R$ is reduced for increasing solar wind velocities. At the same time we expect $\rho$ to increase as well but $D$ to decrease in such a way that $D \rho$ changes only little. At the same time we expect that the natural frequency $\Omega$ will increase with $U$ as well so that the normalized damping constant varies as $\sqrt{R n M / 6 D \rho} \sim U^{-1 / 3}$ by use of Eq. (2).

By numerical simulation we studied also the importance of the simplifying assumption of $\theta=\pi / 2$ in the analysis. Some differences can be noted as already mentioned. Nevertheless 
we find the overall features to be well accounted for by the simple model, even for a case where the magnetic dipole axis is tilted by as much as $\pi / 6$ with respect to the reference case.

We mention also a possibility for a parametrically driven oscillation for cases where the solar wind pressure is fluctuating. This case can be modeled by setting $U=U(t)$ in Eq. (8), recalling that now also $R=R(t)$. If the power spectrum of the solar wind pressure contains significant energy near $\Omega$, we have the possibility of periodic oscillations sustained for a long time. A similar possibility was mentioned also by Kepko and Spence (2003).

Our ambition here was to obtain the simplest possible model, but point out that many details can be added without much additional effort, such as a dilute plasma in the Earth magnetosphere, and a weak solar wind magnetic field. These additions to the model will contribute to the force balance in relation (1). A tilt of the Earth's magnetic dipole axis is readily accounted for, as demonstrated. The model can be generalized to account also for torsional oscillations where the normal of the local plane of the magnetosphere boundary is turning around a line perpendicular to the Sun-Earth direction. In, for instance, Fig. 1a one of the two lines mentioned is vertical in the plane of the figure, the other one perpendicular to this, out of the paper. Thus, two modes can be identified here, one where the line is parallel to the Earth's magnetic dipole axis and one where it is perpendicular.

Acknowledgements. Valuable discussions with Per Even Sandholt are gratefully acknowledged. We thank Liv Larssen from the Auroral Observatory at the University of Troms $\varnothing$ for helping us by preparing Fig. 1. The project was in part supported by the Norwegian National Science Foundation.

Topical Editor I. A. Daglis thanks one anonymous referee for her/his help in evaluating this paper.

\section{References}

Alfvén, H.: Cosmical Electrodynamics, Oxford University Press, London, 1950.

Børve, S., Omang, M., and Trulsen, J.: Regularized smoothed particle hydrodynamics with improved multi-resolution handling, J. Comput. Phys., 208, 345-367, 2005.

Chapman, S. and Bartels, J.: Geomagnetism, Oxford University Press, 1940.

Farrugia, C. J. and Gratton, F. T.: Aspects of magnetopause/magnetosphere response to interplanetary discontinuities, and features of magnetopause Kelvin-Helmholtz waves, J. Atmos. Solar-Terr. Phys., 73, 40-51, doi:10.1016/j.jastp.2009.10.008, 2011.

Freeman, M. P., Freeman, N. C., and Farrugia, C. J.: A linear perturbation analysis of magnetopause motion in the Newton-Busemann limit, Ann. Geophys., 13, 907-918, doi:10.1007/s00585-995-0907-0, 1995.
Gosling, J. T., Thomsen, M. F., Bame, S. J., Onsager, T. G., and Russell, C. T.: The electron edge of low latitude boundary layer during accelerated flow events, Geophys. Res. Lett., 17, 18331836, 1990.

Harrold, B. G. and Samson, J. C.: Standing ULF modes of the magnetosphere: A theory, Geophys. Res. Lett., 19, 1811-1814, doi:10.1029/92GL01802, 1992.

Kepko, L. and Spence, H. E.: Observations of discrete, global magnetospheric oscillations directly driven by solar wind density variations, J. Geophys. Res., 10, 1257, doi:10.1029/2002JA009676, 2003.

Kivelson, M. G., Etcheto, J., and Trotignon, J. G.: Global compressional oscillations of the terrestrial magnetosphere - the evidence and a model, J. Geophys. Res., 89, 9851-9856, 1984.

Korotova, G. I. and Sibeck, D. G.: A case study of transit event motion in the magnetosphere and in the ionosphere, J. Geophys. Res., 100, 35-46, 1995.

Landau, L. D. and Lifshitz, E. M.: Fluid Mechanics, vol. 6 of Course of Theoretical Physics, 2 ed., Butterworth-Heinemann, Great Britain, 1987.

Monaghan, J. J.: Smoothed particle hydrodynamics, Annu. Rev. Astron. Astrophys., 30, 543-574, 1992.

Monaghan, J. J.: Smoothed particle hydrodynamics, Rep. Prog. Phys., 68, 1703-1759, 2005.

Plaschke, F., Glassmeier, K.-H., Sibeck, D. G., Auster, H. U., Constantinescu, O. D., Angelopoulos, V., and Magnes, W.: Magnetopause surface oscillation frequencies at different solar wind conditions, Ann. Geophys., 27, 4521-4532, doi:10.5194/angeo27-4521-2009, 2009.

Samson, J. C., Harrold, B. G., Ruohoniemi, J. M., Greenwald, R. A., and Walker, A. D. M.: Field line resonances associated with MHD waveguides in the magnetosphere, Geophys. Res. Lett., 19, 441-444, doi:10.1029/92GL00116, 1992.

Sibeck, D. G., Baumjohann, W., Elphic, R. C., Fairfield, D. H., Fennell, J. F., Gail, W. B., Lanzerotti, L. J., Lopez, R. E., Lühr, H., Lui, A. T. Y., Maclennan, C. G., Mcentire, R. W., Potemra, T. A., Rosenberg, T. J., and Takahashi, K.: The magnetospheric response to 8-minute period strong-amplitude upstream pressure variations, J. Geophys. Res., 94, 2505-2519, 1989.

Smit, G. R.: Oscillatory motion of the nose region of the magnetopause, J. Geophys. Res., 73, 4990-4993, 1968.

Song, P., Russell, C. T., Gosling, J. T., Thomsen, M., and Elphic, R. C.: Observations of the density profile in the magnetosheath near the stagnation streamline, Geophys. Res. Lett., 17, 20352038, 1990.

Spreiter, J. R., Summers, A. L., and Alksne, A. Y.: Hydromagnetic flow around the magnetosphere, Planet. Space Sci., 14, 223-250, doi:10.1016/0032-0633(66)90124-3, 1966.

Walker, R. J. and Russell, C. T.: Introduction to Space Physics, chap. 6: Solar-wind interactions with magnetized planets, pp. 164-182, Cambridge University Press, Cambridge, UK, 1995. 